\title{
Belphégor
}

\section{Baetens, Jan, The Film Photonovel: A Cultural History of Forgotten Adaptations}

\section{Luca Di Gregorio}

\section{Q OpenEdition}

1 Journals

\section{Electronic version}

URL: https://journals.openedition.org/belphegor/2102

DOI: 10.4000/belphegor.2102

ISSN: 1499-7185

Publisher

LPCM

\section{Electronic reference}

Luca Di Gregorio, "Baetens, Jan, The Film Photonovel: A Cultural History of Forgotten Adaptations", Belphégor [Online], 18-1 | 2020, Online since 14 January 2020, connection on 30 June 2021. URL: http://journals.openedition.org/belphegor/2102 ; DOI: https://doi.org/10.4000/belphegor.2102

This text was automatically generated on 30 June 2021.

\section{(c) (i) (9)}

Belphégor est mis à disposition selon les termes de la Licence Creative Commons Attribution - Pas d'Utilisation Commerciale - Pas de Modification 4.0 International. 


\section{Baetens, Jan, The Film Photonovel: A Cultural History of Forgotten Adaptations}

\section{Luca Di Gregorio}

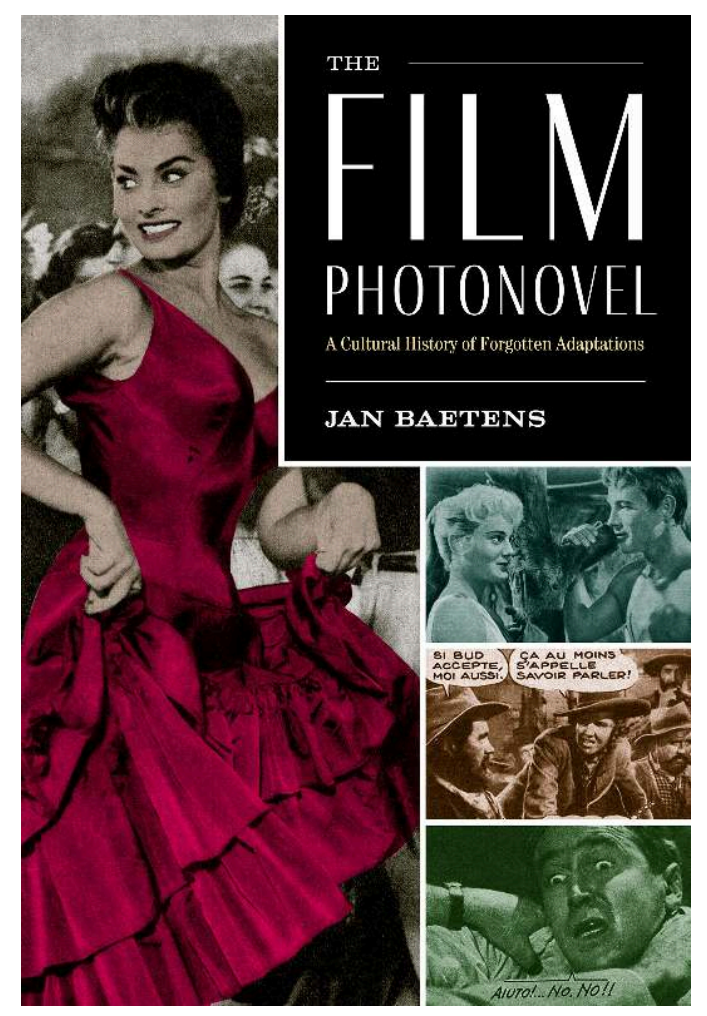

1 Professeur à l'Université de Louvain, Jan Baetens vient marquer d'un nouveau jalon son exploration de longue haleine des genres de la culture médiatique passibles d'une sémiotique à cheval sur l'écrit et l'image - bande dessinée, novellisation, roman-photo et, désormais, ciné-roman-photo. Si c'est ce dernier vocable que Baetens recommande d'employer en français pour désigner le genre ici abordé ${ }^{1}$, son livre, paru aux Presses de 
l'Université du Texas, opte pour film photonovel. La différence est au moins autant affaire de diplomatie conceptuelle que de formalité traductive : de même qu'en italien, les vocables fumetto et fotoromanzo sont minés par une durable polysémie médiatique, en français, un usage traduit de film photonovel aurait requis de bien distinguer l'appellation de l'objet d'une série d'autres, renvoyant à des formes plus ou moins apparentées, et dont la généalogie aurait pu frayer bien en amont. Il aurait en particulier fallu se heurter à l'appellation piégeuse de cinéroman, attestée au moins depuis la Grande Guerre pour désigner ces feuilletons littéraires (novellisations avant la lettre) qui faisaient escorte, dans certains journaux, à la projection de films muets ${ }^{2}$. Pour un premier ouvrage généraliste sur le genre, l'emploi d'une taxinomie anglaise était donc de bon aloi.

2 Par le truchement du film photonovel, Baetens peut faire place nette conceptuelle et entrer de plain-pied dans son analyse d'un genre bien circonscrit, quoique sa présence dans la presse magazine des Trente Glorieuse ait été à la fois abondante, disparate et très variable. Le chapitre II commence par retracer l'histoire transmédiatique du cinéroman-photo en montrant que celui-ci s'est progressivement dégagé de ses voisins proches, que sont la novellisation, le roman dessiné et le roman-photo. On considère généralement qu'il «naît» en 1954, avec quelques années de décalage sur ces deux derniers genres, et se développe comme eux sur le support de la presse magazine italienne. Loin d'être une plate chronologie, le chapitre problématise cette émergence en insistant sur la matrice médiatique commune à toutes ces formes : la démarcation de l'imaginaire cinématographique (au sens large) dans des périodiques populaires ou féminins à dominante mélodramatique. Quand roman dessiné et roman-photo s'adossent au cinéma par l'emprunt indirect de ses atmosphères et de ses figures mythiques, le ciné-roman-photo - plus proche en cela de la novellisation - dépend toujours d'un film-source bien déterminé qu'il s'agit d'adapter - notion largement discutée dans l'ouvrage. En fonction des qualités de sa réalisation, un ciné-romanphoto pourra - au pire des cas - restituer a minima son patron filmique, mais aussi - cas plus heureux - ambitionner de le recomposer, de le raconter à nouveau et à nouveaux frais. Cette pluralité de rapports possibles entre un film-source et son (ou ses) cinéroman(s)-photo(s) induit une marge créative et une latitude sémiotique insoupçonnées. Les chapitres suivants déconstruisent donc la simplicité trompeuse du genre en explorant dans le détail et exemples à l'appui, sa complexité narrative, visuelle et médiatique.

3 Le chapitre III aborde plusieurs aspects de la «bimédialité » du ciné-roman-photo en s'appuyant sur la distinction entre modalités monstrative (showing) et narrative (telling) théorisée par André Gaudreault. Ce duo de notions recoupe de façon assez convaincante le rapport texte-image sur lequel s'appuie inévitablement le genre - et ceci bien que pourraient aussi s'y concevoir des textes monstratifs ou sémaphoriques (une onomatopée, par exemple) ou, comme l'auteur l'envisage plus loin dans l'essai, des images déjà narrantes par leur seule mise en séquence. Reste qu'en tout état de cause, Baetens invite à relativiser l'ascendant qu'une lecture trop rapide attribuerait aux photogrammes (showing) pour souligner, au contraire, la primauté du texte (telling). Dans le ciné-roman-photo, l'écrit prend en charge la conduite de l'essentiel du récit, non seulement au plan quantitatif, par la présence de portions textuelles souvent envahissantes dans l'économie de la page, mais aussi structurellement, la complexité 
narrative d'un film à adapter ne pouvant être rendue par la seule reprise de photogrammes.

4 Faire ce constat de la primauté du narratif permet à l'auteur de poser, en narratologue, une première question au ciné-roman-photo : qui raconte l'histoire ? La réponse l'oblige à concéder d'entrée que, compte tenu de la condition bimédiale et médiatique du genre (articulé sur deux médiums et incrusté dans un média, le magazine), la voix narrative ne saurait y être identifiée dans une seule instance. Ces imbrications et dénotations font en sorte que, dans le ciné-roman-photo, «ça » raconte à plusieurs étages, au point que la distinction même entre le " texte » et son environnement est loin d'être toujours claire. Selon l'auteur, c'est dans les rapports qu'il entretient avec différents niveaux d'épitexte que l'on peut identifier l'acte de raconter, et le récit lui-même. De premiers effets de narration se joueraient ainsi entre le ciné-roman-photo et le " House style » du magazine. Ce «House Style», sorte d'épitexte médiatique, rassemblerait les contenus et rubriques qui résonnent ou se renforcent les unes les autres à l'échelle de toute la publication (éditoriaux de la rédaction, contenus mélodramatiques, rubriques entretenant la liturgie de la vedette, etc.). Le second rapport narratif, plus classique, est interne à l'espace dédié au ciné-roman-photo. Il se donne à voir à ses seuils, dans l'épitexte technique d'ouverture (titre et crédits du film-source, acteurs principaux, etc.) ou dans l'épitexte descriptif qui accompagne le récit (narration textuelle, rappel de l'intrigue, etc.).

5 À cette définition générique des niveaux de narration succède une exploration au grain plus affiné, s'appuyant sur quatre secteurs différents du ciné-roman-photo européen qui, ensemble, donnent à voir la grande variété productive du genre: son modèle classique, le plus souvent imité, représenté par l'éditeur italien Bozzesi ; le modèle des Éditions Mondiales, qui présente la particularité de sérialiser ses ciné-romans-photos (par exemple, dans les feuilletons de Nous Deux Film); le modèle épuré et très lisible de Mon Film, concurrent du précédent, limité au catalogue plus « local » du cinéma français et qui recourt fréquemment à des photographies de tournage ; enfin, le modèle du cinéroman-photo de genre, plus tardif, propose des réalisations hâtives (ex : westerns) qui déséquilibrent l'articulation telling/showing par un choix d'images purement illustratives mais saturées de textes; ces dernières productions, d'ambition ouvertement lowbrow, se passent souvent de renseigner le film-source.

6 Les chapitres IV et $\mathrm{V}$ abordent la composition formelle du ciné-photo-roman à travers les contraintes posées par le médium. Le chapitre IV, axé sur le framing, commence par relativiser les analogies que l'on peut faire entre les procédés de composition du cinéroman-photo et ceux des genres qui lui sont proches. Baetens distingue d'abord sa mise en page du montage cinématographique : il fait ainsi valoir que le ciné-roman-photo doit composer, non seulement avec le récit déroulé case après case, mais aussi avec l'espace de la page, qui demeure l'unité expressive traditionnelle des littératures graphiques. La scansion (dans le temps) des photogrammes doit dès lors aussi s'articuler à la distribution (spatiale) de tableaux de six cases (presque toujours 3X2), qui sont autant d'« écrans » éclatés sur chaque page. D'autres analogies, comme celle qui nous porterait à rapprocher le framing du ciné-roman-photo à celui du romanphoto et de la bande dessinée, ne tournent pas moins court aux yeux de l'auteur : ici, le hiatus semble inversé par rapport à l'analogie du montage cinématographique, puisque c'est le protocole d'adaptation d'un film-source qui revient contraindre la composition. Quand bande dessinée et roman-photo, en toute hypothèse, peuvent déployer 
«librement » leur histoire, le ciné-roman-photo, lui, doit démarquer un film-source depuis son flux d'images parlantes. Ce film devra être rendu doublement lisible: lisible dans sa lecture linéaire et séquentielle, mais aussi dans celle, tabulaire, qu'induit l'espace unitaire de la page - d'où le rôle stratégique assigné aux «coins » des pages assurant les transitions visuelles, ou aux fréquents jeux de lecture "par colonnes». Une autre contrainte de composition est induite par le média du magazine. Celui-ci pourra prescrire, selon les cas, une surreprésentation de l'image de la star, un style léché lorsqu'il s'agit d'adapter (notamment en France) des films à valeur nationale ou patrimoniale, ou encore une contrainte d'irrégularité de présentation de la page. Le repérage de cette contrainte permet à Baetens d'expliquer la faible lisibilité de certains ciné-romans-photos par la nature du média qui les supporte: ainsi, dans les productions d'Amor Film, l'anarchique mise en page apparaît comme symptomatique d'un magazine qui hésite encore entre le ciné-roman-photo et son ancienne spécialisation dans la novellisation.

7 Dans le chapitre V, la contrainte examinée concerne la restitution de l'action et du mouvement des films-sources par les ciné-romans-photos. Partant du jugement courant selon lequel la fixité du photogramme constituerait un obstacle rédhibitoire à la pleine adaptation du film, l'auteur invite à un déplacement de perspective. Le mouvement, tel qu'exprimé au cinéma, n'est pas la jauge absolue d'une adaptation photo-romanesque. Au contraire, les ciné-photo-romans les plus réussis renoncent souvent à rendre l'action en prise directe avec son régime filmique - entreprise en effet périlleuse - pour privilégier, quitte à trahir un peu, un redéploiement, une recomposition pensée depuis les ressources propres au médium. Plusieurs stratégies obliques concourant à cet effet sont examinées : la substitution du mouvement par une scénarisation de ses effets; la recherche d'un autre type d'induction de la tension narrative, notamment grâce au recentrage de l'action (l'analyse de l'adaptation « intelligemment infidèle » de Fenêtre sur cour d'Hitchcock est en cela particulièrement convaincante); un usage suggestivement dynamique de la planche composée, de la répétition d'images identiques ou recadrées, ou encore un travail sur les poses, conçues comme des tableaux vivants. Sans reproduire directement ni intégralement ce qui animait le film, ces expédients, exemples à l'appui, paraissent en effet en mesure de fonder ou de remotiver l'action en s'appuyant sur d'autres effets - voire, d'autres affects: dans ses quelques pages consacrées à l'ambivalence des scènes de danse, Baetens monte notamment comment celles-ci, pourtant stérilisées de toute chorégraphie, ont su tirer profit de leur statisme pour «prendre la pose » et demeurer un moment attendu - et iconique - du ciné-photo-roman.

8 Le dernier chapitre (VI) n'est pas tant une conclusion qu'une ouverture sur la fortune mondiale du ciné-roman-photo (Amérique latine, Afrique du Sud, États-Unis, etc.) à laquelle succède une autre ouverture, sous forme d'hommage, au film La Jetée (1962) de Chris Marker. En mettant l'accent sur cet aérolithe - œuvre expérimentale et hors circuit médiatique qui narre une histoire au moyen d'une suite de clichés immobiles mais que Marker considérait comme un "photo-roman"-, Baetens attire plus fondamentalement l'attention sur les potentialités d'une recherche narrative combinant les médiums du cinéma et des images fixes. Au risque, peut-être, de trancher un peu brusquement avec le pari du " genre à contraintes » sur lequel misait l'argumentation qui précédait, qui considérait le ciné-roman-photo dans les déterminations de sa formule médiatique et commerciale d'origine. 
9 The Film Photonovel: A Cultural History of Forgotten Adaptations marque un effort majeur pour rendre compte de la complexité d'un genre oublié et méprisé (sans doute plus que le roman-photo lui-même), tandis que son originalité de méthode tient au choix, soutenu tout au long du livre, d'élever ce genre au sérieux d'une scrupuleuse étude sémiotique. Cette sémiotique à laquelle appelle le ciné-roman-photo étant multimodale et médiatiquement intégrée, elle engage plusieurs dimensions que Jan Baetens affronte pied-à-pied, dans des chapitres qui mordent les uns sur les autres et s'agencent en panoptique, parcourant alternativement les niveaux de dénotation et de signification du genre, ses contrastes intermédiaux et sa créativité sous contraintes. À cet effet, la présence d'une grande variété d'exemples choisis avec grand soin constitue l'atoutmaître d'une argumentation dont les raffinements, grâce à cet appui, collent véritablement au genre, sans réduire celui-ci à un simple prétexte pour expérimentations de sémiologie médiatique hardcore. Lorsqu'il théorise, l'auteur le fait aussi en encyclopédiste. Son corpus considérable l'autorise à creuser très en détail l'analyse de faits de sens qui étaient loin d'aller de soi, n'hésitant pas à étudier, par moments, jusqu'à trois ciné-romans-photos différents adaptant le même film. Le pari de l'essai nous en paraît doublement réussi : non seulement parce qu'y est démontré l'intérêt d'une lecture très rapprochée du ciné-roman-photo, couvrant presque tous les aspects de sa production et de sa composition ; mais aussi parce que pareille lecture dans un second temps - contribuera grandement à une réflexion d'ordre plus général sur l'adaptation et la transmédiatisation.

10 Un dernier mérite, moins central mais pas si indifférent, de cette méthode étayée par laquelle Jan Baetens nous interpelle sur la complexité d'un genre aussi déprécié, est que son pouvoir de conviction n'a pas besoin de recourir au langage de la " réhabilitation discursive " d'un "mauvais genre " que l'air du temps donnait à craindre. L'essai n'investit pas dans ces réhabilitations externes, plus revanchardes qu'éclairantes, et qui se satisfont trop exclusivement de déconstruire les griefs socioculturels imputés à n'importe quelle production de masse. Pourtant, dans la plénitude même de son propos, l'auteur parvient d'autant mieux - nous semble-t-il - à intéresser en profondeur au ciné-roman-photo. Parce qu'il prend son objet au sérieux sans plaider le "malentendu historique ", mais aussi - selon ses propres dichotomies - parce que son telling critique est un showing permanent, le propos se déroule de telle sorte que le procès de réhabilitation pourra émaner du lecteur lui-même, par l'intelligence positive qu'il aura conçue de l'objet après avoir refermé le livre.

11 Bien sûr, l'utilisation quelque peu adaptée que propose l'auteur de certains outils classiques empruntés, notamment, à la narratologie, pourrait lever ici ou là quelques objections ; même si ces objections, ici encore, appellent moins la polémique qu'une discussion encore stimulante pour la réflexion. Par exemple, il nous semble discutable de faire figurer sur un même plan, au titre de la "voix narrative" (chap. III), le véritable rapport de narration qu'induit l'épitexte descriptif interne au ciné-romanphoto, et des effets d'énonciation éditoriale (É. Souchier) plus diffus, émanant d'instances connexes - "House Style ", épitexte médiatique - par lesquelles le ciné-roman-photo est lié au reste du magazine. Ces derniers effets seraient plutôt des produits par recoupement d'un ethos de presse : s'ils disent et orientent effectivement une identité de surplomb, ils le font sans prendre strictement en charge un "discours du récit " dans le ciné-roman-photo. En cela, ils seraient moins vecteurs de narrativité qu'adjuvants de ce que Marie-Ève Thérenty appelle la « poétique du support ${ }^{3}$ ». 
facteurs que gagnerait à recontextualiser une perspective qui se montrerait également attentive à la réception, et pour mieux dire aux pratiques (populaires) ${ }^{4} \mathrm{du}$ ciné-romanphoto, lesquelles ont souvent dévié des lectures programmées dans l'amont de la composition. Ainsi, là où Baetens souligne le rôle primordial du texte et de l'écrit dans la conduite du récit et la recomposition du film-source, il est fort à parier qu'une étude de l'acculturation à « l'objet film photonovel » par ses publics des Trente Glorieuses ferait apparaitre la prééminence de lectures plus sélectives, où le visuel pourrait bien récupérer la main, voire devenir hégémonique, notamment à travers les poses et instantanés de cinéma que le ciné-roman-photo offrait à redécouvrir, à collecter, à posséder dans le cadre domestique. Il est vrai toutefois que ce type d'approche jouxtant l'histoire culturelle a déjà été ébauché pour le domaine italien ${ }^{5}$, tandis que l'approche sémiotique de Baetens demeurait largement inappliquée au genre.

Ces deux approches, répétons-le, n'ont rien d'incompatible, et paraissent tout à fait articulables au sein de travaux où l'histoire culturelle ne ferait pas pièce à la discussion des formes - et vice versa. L'une des intersections possibles résiderait précisément dans cette question des pratiques populaires du cinéma telles que celles-ci anticipaient, dans leur déviation de l'expérience filmique, ce qu'ensuite roman-photo et ciné-romanphoto donneront à lire, dans leurs configurations formelles et visuelles bien déterminées. Dans son "Autobiographie d'un spectateur", Italo Calvino se rappelle ainsi avoir été marqué par la forme d'irrespect créateur que manifestaient les habitués des salles de cinéma de son enfance :

On peut dire que déjà à cette époque nous parcourions les techniques narratives les plus sophistiquées du cinéma d'aujourd'hui, en cassant le fil temporel de l'histoire et en la transformant en un puzzle qu'il fallait recomposer morceau par morceau ou accepter sous la forme d'un corpus fragmentaire ${ }^{6}$.

14 N'est-il pas envisageable dès lors que cette pratique déviante, largement attestée dans l'acculturation populaire au film, fût en un sens déjà photo-romanesque; et qu'en guettant la meilleure pose des vedettes, en découpant des détails dans le cadre et des instantanés dans l'image animée, le spectateur populaire ait institué, par sa propre fréquentation du film, un régime sous-jacent du regard, qui aurait sollicité et préparé de lui-même l'avènement de ces premiers genres du Home Entertainment, de la pratique domestique du cinéma ? Pareille prise en compte des ciné-romans-photos - mettons comme des péritextes autonomes faisant transiter la pratique du film dans le foyer (avant la démocratisation de la T.V.), dégagerait peut-être un terrain médian, où la composition du genre, sa sémiotique, pourraient se voir remotivées à la lumière des évolutions conjointes de l'histoire culturelle et de la culture de masse.

\section{NOTES}

1. C'est ce terme qu'il utilise, par exemple, dans un récent article publié en français: «Jan Baetens, Ascenseur pour l'échafaud en images fixes, Louis Malle en roman-photo ", dans Études françaises, n. 55 (2), 2009, pp. 57-73. 
2. On trouvera dans un numéro récent de la revue Le Rocambole justement consacré au cinéroman (n. 78/79, juin 2017) un examen extensif de ce « proto-photo-roman».

3. Marie-Ève Thérenty, « Pour une poétique du support », Romantisme, n. 43, 2019/01, p. 109-115.

4. Et nous pensons bien sûr ici aux « arts de faire » de Michel de Certeau.

5. Représentatif de ce genre d'approche, citons un autre ouvrage tout juste paru, et où les rapports culturels entre roman-photo et cinéma sont mis en perspective grâce aux travaux de Michel De Certeau: Silvana Turzio, Il fotoromanzo: Metamorfosi delle storie lacrimevoli, Milan, Meltemi, 2019.

6. Italo Calvino, La Route de San Giovanni, Paris, Gallimard, « Folio », 2018, p. 57.

\section{AUTHOR}

\section{LUCA DI GREGORIO}

Luca Di Gregorio est docteur en lettres à l'Université de Liège. Ses recherches interrogent principalement les imaginaires européens de l'Ouest américain, selon une perspective articulant les outils de la critique culturelle et de la littérature comparée avec certaines dimensions de la production populaire et de masse. Auteur de Wilderness et Western. L'Ouest fictionnel chez Gustave Aimard et Emilio Salgari (Presses Universitaires de Liège, 2014), il s'est aussi intéressé, dans plusieurs articles, à des figures populaires comme Buffalo Bill, le trappeur ou l'Indian-hater, ou au genre du roman de détective ethno-écologique dans des travaux publiés par Garnier, Routledge ou les revues Belphégor, Ilcorsaronero. 